\title{
US Office of Naval Research celebrates 75 years
}

$\mathrm{T}^{\mathrm{T}}$ The US Office of Naval Research (ONR) held a virtual event, "ONR at 75: Reimagine Naval Power," on Sept. 30, 2021. Established in 1946, ONR-sponsored science and technology has laid the foundation for the first digital, real-time computing systems; invested in early groundbreaking research in artificial intelligence and directed energy; and helped create the first autonomous vehicles and robotic systems.

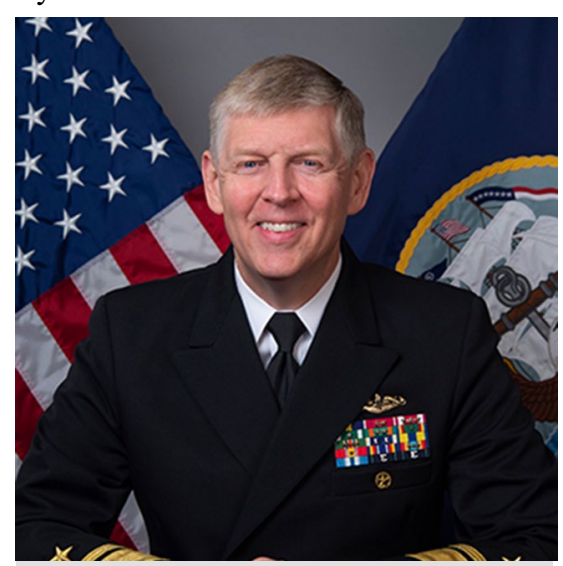

Rear Adm. Lorin C. Selby

Chief of Naval Research

In his keynote address, Rear Admiral Lorin C. Selby, who became Chief of Naval Research in May 2020 , said that the future is driven by technology and by economic change. The future, he said, "is not just about things, not just about devices. It's also about process. It's about STEM [science-technology-engineeringmathematics]. It's about how do we educate the next generation of leaders." He said it is necessary to make sure "that it's a very diverse crowd of individuals who want to pursue STEM careers."

Selby himself was sparked to pursue a STEM career as a child when he saw the first astronaut walk on the moon. In college, he was further inspired by his materials science professor who was conducting research for ONR on corrosion on a submarine. The professor showed him "how we're seeing all kinds of issues going on with dissimilar metals." Selby eventually pursued nuclear engineering, earning a BS degree from the University of Virginia and an MS degree from the Massachusetts Institute of Technology.

When Selby was sworn in as Chief of Naval Research, according to a news release, he said, "ONR is rightly celebrated as the Navy's leading science and technology organization. I hope to build on that legacy by stimulating collaboration and partnerships that will enable our warfighters to tackle the challenges of 21st-century warfare." As part of the 75th anniversary event, he introduced and moderated a panel to discuss the future of warfare and its connections to research.

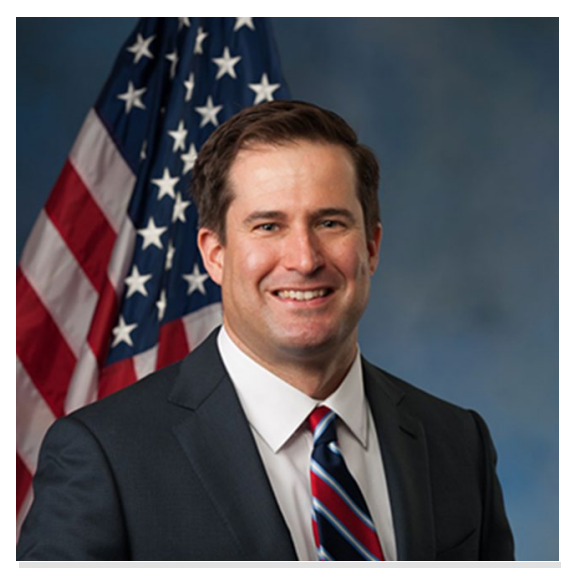

Rep. Seth Moulton (D-Mass.)

House Armed Services Committee

The panelists-Representatives Seth Moulton (D-Mass.) and Mike Waltz (R-Fla.), serving on the US House Armed Services Committee; Brig. Gen. Benjamin Watson, Vice
Chief of Naval Research; and Dr. Jason Stack, ONR Director for Ocean, Atmosphere and Space Research-emphasized two main changes in the ONR approach to $\mathrm{R} \& \mathrm{D}$. One is a partnership with industry and academia, the other is a new type of global partnership.

Moulton explained that a change in ONR practice from the 1950 s is its need to partner with industry and academia. "We're still stuck in the 1950 s where we think everything is going to be developed by government," he said, "and what we really need to do is tap into the private

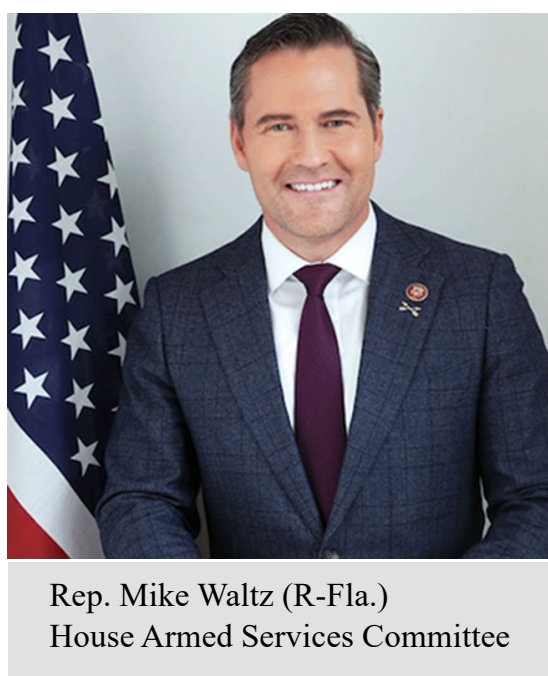

sector, to make sure the private sector is adequately able to get the technology to the services." Selby agreed, suggesting the need for the military to adapt technology available from industry, or to define their problem to industry for solutions.

At the same time, Moulton acknowledged that some of the major technological challenges are in areas such as biotechnology to create devices. "It's very cutting edge, super risky," he said, "research risks that the private sector is not necessarily willing to take on." He does not want to pull funding away from other government agencies that fund that kind of research, 
but he commented that the armed forces need to reform the acquisitions process.

Waltz agrees, stating, "We just reauthorized NSF [the National Science Foundation] which is responsible for $30-40 \%$ of basic and applied research that goes on in our universities." Waltz is also very interested in autonomous systems where humans are the slowest and weakest link. "The next evolution is when we pair systems with artificial intelligence," he said. To conduct R\&D on advanced systems such as artificial intelligence and quantum, the United States needs to maintain access to rare-earth elements, he said. Along with Moulton, Waltz is disappointed with current US policy regarding Afghanistan, which holds the second largest known lithium reserves, the third largest known copper, and fifth largest known cobalt reserves. "All of which we need to have access to readily before we can even start talking about how we integrate autonomous [systems]," he said.

Introducing the topic of international collaborations, Selby talked about technology being developed around the world. He said, "The United States should be their partner of choice when it comes to science and technology." Moulton responded that previous alliances were built on region, such as NATO. The new generation of alliances should be based on technology with leading technology nations like the US, Israel, and Norway. Waltz acknowledged the caveat that within these alliances, technology protocols and legislation should be in place to prevent research theft and fraud. From the military side, Stack said, "There are actually disproportionate advantages that we get" from keeping science and research open with our international partnerships.

The panel drew to a close with a discussion on STEM careers. Waltz said that the STEM community includes just $11 \%$ minorities and 25-27\% women. More research is needed, he said, to understand how to increase these percentages. From the military perspective, Watson agrees: "The Marine Corps need to change the demographics of our workforce, of our people ... to be consistent with the technological sophistication of the equipment with which we are manning them." He praised the emphasis on people when reimagining the future, stating that young women and men can be encouraged to serve national security through STEM.

Judy Meiksin

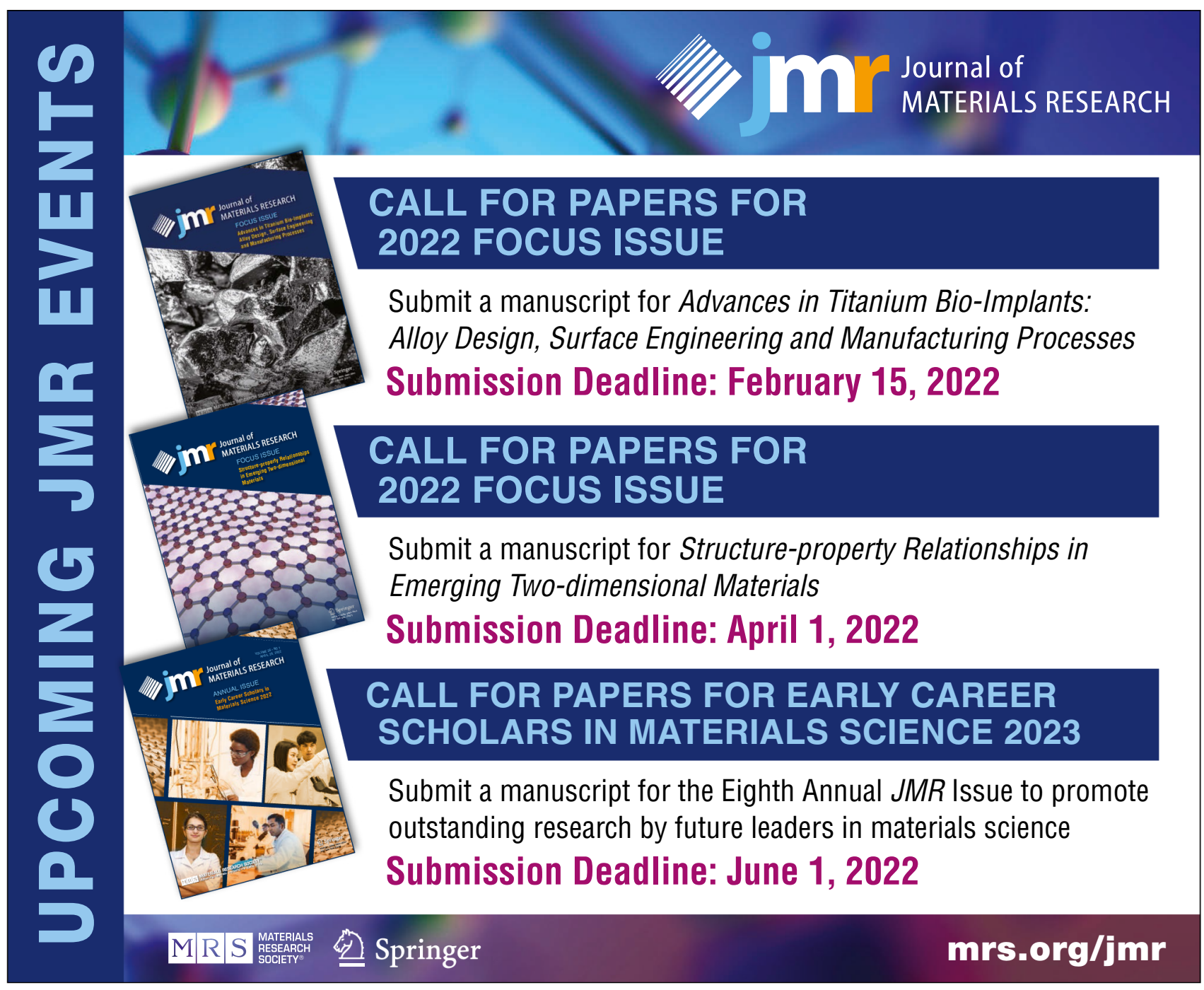

